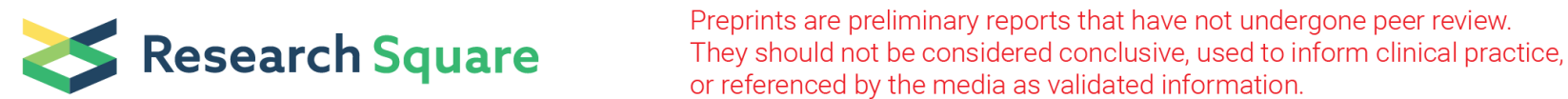

\section{Pilot Study of Repeated Blood-Brain Barrier Disruption in Patients with Mild Alzheimer's Disease with an Implantable Ultrasound Device}

\section{Stephane Epelbaum}

Hôpital Pitié-Salpêtrière https://orcid.org/0000-0003-4059-2891

Ninon Burgos

Institut du cerveau (ICM)

Michael Canney

Carthera

Dawn Matthews

Admdx

Marion Houot

AP-HP: Assistance Publique - Hopitaux de Paris

Mathieu D. Santin

Institut du cerveau (ICM)

Carole Desseaux

Carthera

Guillaume Bouchoux

Carthera

\section{Sebastian Stroer}

APHP: Assistance Publique - Hopitaux de Paris

Cyril Martin

Carthera

Marie-Odile Habert

APHP: Assistance Publique - Hopitaux de Paris

Marcel Levy

APHP: Assistance Publique - Hopitaux de Paris

Aicha Bah

APHP: Assistance Publique - Hopitaux de Paris

Karine Martin

APHP: Assistance Publique - Hopitaux de Paris

\section{Benoît Delatour}

Institut du cerveau (ICM)

Maximilien Riche 
APHP: Assistance Publique - Hopitaux de Paris

\section{Bruno Dubois}

APHP: Assistance Publique - Hopitaux de Paris

\section{Lisa Belin}

APHP: Assistance Publique - Hopitaux de Paris

Alexandre Carpentier ( $\sim$ alexandre.carpentier@aphp.fr )

Sorbonne Université

\section{Research}

Keywords: Alzheimer's Disease, Clinical Trial, Ultrasound, Florbetapir, Amyloid, Position emission tomography, magnetic resonance imaging, blood-brain barrier

Posted Date: October 25th, 2021

DOl: https://doi.org/10.21203/rs.3.rs-965161/v1

License: (c) (1) This work is licensed under a Creative Commons Attribution 4.0 International License. Read Full License

Version of Record: A version of this preprint was published at Alzheimer's Research \&amp; Therapy on March 8th, 2022. See the published version at https://doi.org/10.1186/s13195-022-00981-1. 


\section{Abstract}

Background: Temporary disruption of the blood-brain barrier (BBB) using pulsed ultrasound leads to the clearance of both amyloid and tau from the brain, increased neurogenesis, and mitigation of cognitive decline in pre-clinical models of Alzheimer's disease (AD) while also increasing BBB penetration of therapeutic antibodies. The goal of this pilot clinical trial was to investigate the safety and the efficacy of this approach in patients with mild AD using an implantable ultrasound device.

Methods: An implantable, $1 \mathrm{MHz}$ ultrasound device (SonoCloud-1) was extradurally implanted under local anesthesia in the skull of 10 mild AD patients to target the left supra-marginal gyrus. Over 3.5 months, seven ultrasound sessions in combination with intravenous infusion of microbubbles were performed twice per month to temporarily disrupt the BBB. ${ }^{18} \mathrm{~F}$-Florbetapir and ${ }^{18} \mathrm{~F}$-fluorodeoxyglucose positron emission tomography (PET) imaging were performed on a combined PET/MRI scanner at inclusion and at four and eight months after initiation of sonications to monitor brain metabolism and amyloid levels along with cognitive evaluations. Evolution of cognitive and neuroimaging features were compared to that of a matched sample of control participants.

Results: A total of 63 BBB opening procedures were performed in nine subjects. The procedure was welltolerated. A non-significant decrease in amyloid accumulation at four months of $-6.6 \%(\mathrm{SD}=7.2 \%)$ on ${ }^{18} \mathrm{~F}$ Florbetapir PET imaging in the sonicated gray matter targeted by the ultrasound transducer was observed compared to baseline in six subjects that completed treatments and who had evaluable imaging scans. No differences in longitudinal change in glucose metabolism were observed compared to neighboring or contralateral regions or to the change observed in the same region in ADNI participants. No significant effect on cognition evolution was observed in comparison to the ADNI participants as expected due to the small sample size and duration of the trial.

Conclusions: These results demonstrate the safety of ultrasound-based BBB disruption and potential of this technology to be used as a therapy for $A D$ patients. They support further research of this technique in a larger clinical trial with a device designed to sonicate larger volumes of tissue and in combination with disease modifying drugs to further enhance the effects observed.

Trial registration: NCT03119961 (clinicaltrials.gov)

\section{Background}

Alzheimer's disease (AD) is a growing global health concern with an annual incidence of 1.8 million people in the USA and Europe (1). Although an understanding of the underlying pathophysiology of the disease has grown over the past several decades, no effective treatments exist that slow cognitive decline.

$A D$ is characterized by an accumulation of $\beta$-amyloid in plaques and neurofibrillary tangles composed of tau in the brain. Both $\beta$-amyloid and tau have been the targets of extensive drug development (1), with 
aducanumab, a human monoclonal antibody that selectively binds to $\beta$-amyloid fibrils and soluble oligomers, provisionally approved by the Food and Drug Administration in the United States in $2021(2,3)$. However, such treatments' effect on amyloid load and cognitive function are dose dependent (4) and only $0.1 \%$ of intravenously injected anti-Aß immunoglobulins reach the brain despite a half-life of $15-20$ days (5).

The poor penetration of current antibody therapies for $A D$ is due to their large size ( $>150 \mathrm{kDa}$ ) and the presence of the blood-brain barrier (BBB), which limits $98 \%$ of small $(<500 \mathrm{Da})$ and almost $100 \%$ of larger ( $>500 \mathrm{Da}$ ) molecules from entering the brain parenchyma (6). Thus, there is a need to improve the bioavailability of these antibodies in the brain to improve their efficacy.

The use of low intensity pulsed ultrasound in combination with systemic injection of microbubbles has been explored for the past two decades as a method to temporarily disrupt the BBB (7). Ultrasound-based blood-brain barrier disruption (US-BBBD) allows for increased penetration of systemically administered small and large molecule drug therapies into the brain (8-10). Strikingly, US-BBBD alone has been shown to reduce $\beta$-amyloid and tau pathologies, stimulate neurogenesis, and improve cognitive performance in mouse AD models (11-13) and can be further coupled with drug therapies to improve their brain penetration and efficacy $(8,14,15)$.

Clinical-stage devices have been developed using extracranial $(16)$ and implantable $(17,18)$ approaches. These devices are being tested in clinical trials in patients with brain tumors $(18,19)$ and neurodegenerative diseases $(20,21)$. Recently, the safe disruption of the BBB in six AD patients using a transcranial focused ultrasound system was demonstrated $(22,23)$.

The goal of this phase $1 / 2$ clinical trial was to test the hypothesis that US-BBBD using an implantable ultrasound device is safe in early AD patients and could lead to a reduction in $\beta$-amyloid pathology when used alone. Seven repeated sonications every two weeks were performed during the first four months after study inclusion to disrupt the BBB. Positron emission tomography (PET), magnetic resonance imaging (MRI), and cognitive assessments were used to monitor treatments and disease progression at 0 , 4 , and 8 months after inclusion.

\section{Methods}

\section{Study Design}

This investigator-sponsored phase I/II trial was a single center, exploratory clinical trial (NCT03119961) initiated at Hôpital de la Pitié Salpêtrière (Paris, France). The primary objective was to evaluate changes on PET imaging on $\beta$-amyloid and glucose in the region of interest (ROI) targeted by the ultrasound device.. Secondary objectives were to assess the radiographic and clinical tolerance of repeated BBB opening by ultrasound and to examine the opening of the BBB on T1-weighted (T1w) MRI, and to study the evolution of cognitive decline. The study was approved by the Paris VI Ethical Committee. Informed consent was obtained from all participants. 
Patients between the ages of 50-85, with early-stage AD (mini-mental state examination [MMSE] 20-26) were eligible. Inclusion was based on cognitive assessment (24) and an MRI showing one of the three most frequent phenotypic presentations of the disease (hippocampal amnesia, logopenic aphasia, or posterior cortical atrophy syndrome). Diagnosis was confirmed by the presence of cerebrospinal fluid levels of ptau/Aß1-42 > 0.11 (25).

No control subjects were included in the study, but 45 controls were sampled from the Alzheimer's Disease Neuroimaging Initiative (ADNI) database (adni.loni.usc.edu) through a matching procedure taking into account age, gender, MMSE score and diagnosis (mild cognitive impairment/AD).

\section{Ultrasound device}

The SonoCloud-1 implantable ultrasound device (CarThera, Paris, France) was used for sonications (Figure 1). This investigational device was previously used in a Phase 1/2a study in patients with recurrent glioblastoma who had monthly repeated ultrasound-mediated BBB opening prior to receiving carboplatin chemotherapy $(17,18)$. The device consisted of a 10-mm diameter, $1 \mathrm{MHz}$ ultrasound implant encapsulated in a biocompatible housing. The device was placed in a 12-mm diameter burr hole in the left parietotemporal junction targeting the left supramarginal gyrus using a neuronavigation system under local anesthesia. To activate the device, it was connected using a transdermal needle to a radiofrequency generator, with the first activation occurring at least 15 days after device implantation. During sonications, a 25,000-cycle pulse was used every second for a duration of four minutes in combination with intravenous injection of SonoVue ${ }^{\circledR}$ microbubbles $(0.1 \mathrm{~mL} / \mathrm{kg}$, Bracco). The device was activated every two weeks over the course of seven sessions after patient inclusion ( 3.5 months) The acoustic pressure, initially set at $0.9 \mathrm{MPa}$ was increased after the first sonication session to $1.03 \mathrm{MPa}$. At nine months after implantation, the device was explanted.

\section{Safety Assessments}

Safety assessments included physical and neurologic examinations, and the collection of adverse-event (AE) data according to the Common Terminology Criteria for Adverse Events (CTCAE) v4.0.

\section{Cognitive Evaluation}

The neuropsychological evaluations were performed at baseline, four months and eight months. The evaluation comprised the MMSE (26), Clinical Dementia Rating Scale Sum of Boxes score (CDR-SB) $(27,28)$, Frontal assessment battery (FAB) $(29)$, the free and cued selective reminding test (FCSRT) (30), trail making test (TMT) (31), verbal fluencies (32), praxis (33), Rey's figure (34), State-Trait Anxiety Inventory: STAI (Form Y) (35) and Montgomery-Åsberg Depression Rating Scale (MADRS) (36).

\section{MRI/PET Imaging Acquisition}

MRI imaging was performed following the BBB opening procedure during the first and third sessions on a 3T Prisma Fit (Siemens, Erlangen, Germany) using a 64-channel head coil for signal reception. T2-FLAIR weighted images ( $1 \mathrm{~mm}$ isovoxel) and diffusion weighted images ( $2 \mathrm{~mm}$ isovoxel) were acquired for 
monitoring for any potential edema induced by the BBB disruption procedure. Quantitative susceptibility mapping images ( $1 \mathrm{~mm}$ isovoxel) were obtained using multi-echo $\mathrm{T} 2^{*}$-weighted images to detect any potential hemorrhages. To evaluate BBB disruption, T1 maps (1 mm isovoxel) were then obtained with the MP2RAGE sequence before and seven minutes after a bolus injection of $0.2 \mathrm{~mL} / \mathrm{kg}$ gadolinium-based contrast agent (Gd-DOTA, DOTAREM, Guerbet, France). These images were planned to be performed 60 minutes after sonication.

PET imaging to examine amyloid and glucose in the brain was performed at 0,4 , and 8 months after subject inclusion. PET acquisitions were performed on the PET/MR SIGNA 3T system (GE Healthcare) after implantation of the device. The two acquisitions took place 48 hours apart. Amyloid PET imaging started 50 minutes after intravenous injection of $370 \mathrm{MBq}$ of ${ }^{18} \mathrm{~F}$-Florbetapir and FDG PET imaging started 30 minutes after intravenous injection of $2 \mathrm{MBq} / \mathrm{kg}$ of ${ }^{18} \mathrm{~F}$-fluorodeoxyglucose (FDG). During the period of FDG tracer uptake, participants were at rest with eyes open but ears closed to minimize MRI scanner noise. The 50-minute post-injection start time for amyloid PET was used to maximize a pseudoequilibrium state. For both radiotracers, acquisition parameters were as followed: simultaneous PET/MRI acquisition with i) 20-minute PET acquisition ii) acquisition of four T1 DIXON sequences: in-phase, opposed-phase, fat-only, and water-only and a zero echo time sequence to capture bone information (37); the five images are combined to create a $\mu$ map, used for attenuation correction of the images. iii) $3 D$ T1w anatomical sequence.

\section{BBB disruption efficiency}

To evaluate BBB disruption efficacy, the map of Gd-DOTA concentration was calculated from the difference of the registered T1 maps, considering a T1 relaxivity of $4.5 \mathrm{mM}^{-1} . \mathrm{s}^{-1}$ (38). As a metric for BBB disruption efficacy, the total quantity of Gd-DOTA in sonicated brain tissues was calculated in a $15 \times 55 \mathrm{~mm}$ cylindrical ROI covering the ultrasound beam generated by the implant and compared with the Gd-DOTA quantity in a symmetric contralateral control ROI (Supp Fig. 3). The volume of brain voxels with an enhanced concentration of Gd-DOTA was also calculated in the ROI, using a concentration threshold automatically adjusted such that less than $5 \%$ of the control ROI was classified as enhanced. An ultrasound-mediated BBB opening was considered successful if the quantity of Gd-DOTA in the ROI was greater than the quantity in the symmetric control ROI plus two standard deviations of all control ROls. BBB opening was also visually assessed as in our previous study (17).

\section{Image processing}

Scans (timeframes already averaged for PET) were visually inspected for anatomical completeness, subject motion, and other artifacts, and converted to NIFTI format. MRI scans $(0.488 \times 0.488 \times 1.2 \mathrm{~mm})$ were resliced to $1 \times 1 \times 1 \mathrm{~mm}$ and processed using Corlnsights MRI, which uses Freesurfer 6.0 and other algorithms for segmentation. PET scans were co-registered to their corresponding resliced volumetric MRI scans as produced by Freesurfer. PET scans obtained at four and eight months were additionally 
coregistered to their corresponding initial scans. Baseline MRI scans were spatially transformed to template space using SPM12, and the transformation applied to the co-registered PET scans.

\section{Amyloid PET analysis}

To confirm the presence of amyloid at baseline and to assess longitudinal changes in brain regions that are typically amyloid positive in Alzheimer's disease, values were measured in posterior cingulate, precuneus, lateral temporal, frontal, and anterior cingulate regions. SUVRs were evaluated using whole cerebellum and eroded subcortical white matter as comparative reference regions. SUVRs in global cortex and a relatively large temporoparietal region were also measured using additional processing and reference region approaches as described under Additional Analyses below. A visual read was also performed at baseline.

To evaluate local sonication effects, a custom volume of interest was created for each participant centered at the implant location and extending inward approximately perpendicular to the skull at the position of the implant, with initial dimensions of $10 \times 10 \times 40 \mathrm{~mm}^{3}$. A thresholded version of each volume was created to eliminate cerebrospinal fluid (CSF) from the measured boundaries. Additional custom volumes of interest were created to measure distal tissue in the same coronal slices within the same hemisphere, as well as in the opposite hemisphere, serving as comparator ROls (Figure 2). The ROIs local to the implant were additionally restricted to include only gray matter to assess the effects of including white matter (which provided a slightly larger ROI less vulnerable to technical or motion-related variability) upon measured values.

Standardized Uptake Value Ratios (SUVRs) were calculated as the ratio of the value in the implant ROI divided by the value in each of the comparator ROIs as the reference (same hemisphere and opposite hemisphere, separately). This approach minimized technical variability that can arise from using a reference region located in distant slices of the brain, while maximizing similarities in tissue kinetics. SUVRs were also calculated relative to the overall bilateral parietal region. This provided a comparison to similar tissue at a similar general spatial location within the brain but with larger volume to reduce technical noise. Whole cerebellum and white matter were also evaluated as reference regions but this was for information only given technical noise associated with the cerebellar reference in longitudinal measurement (39) and the better spatial and tissue type match obtained using adjacent and opposite hemisphere tissue as the reference.

The reliability of the target region amyloid measures for each subject was assessed by determining whether unacceptable embedded head motion had occurred during the scan. Motion would be indicated by spiral artifact in the MRI scan (acquired in the same session and position as the PET scan) and/or by longitudinal change in regions distant from the implant that were well beyond the range expected over 4 and 8 months physiologically based upon numerous studies (39).

\section{FDG PET analysis}


FDG PET scans were evaluated using the same standard and custom ROls and reference region approaches that were defined for analysis of the amyloid scans. In addition, voxel-based multivariate machine learning classification software was applied to explore patterns discriminating baseline, month 4 (M4), and month 8 (M8) states. Briefly, the spatially normalized, smoothed FDG scans for the three timepoints were grouped into three $(\mathrm{N})$ training classes. Using the NPAIRS software framework, principal components (PCs) were determined for the data set, after which canonical variates analysis (CVA, a form of linear discriminant analysis) was used to mathematically combine the most significant PCs into N-1 (two) patterns of hypometabolism and hypermetabolism or preservation relative to whole brain. The data set was split into halves numerous times, each time using each half to generate a model (patterns) and generating a reproducibility metric as the correlation between the patterns, and a prediction metric based on the classification of one half from the other half's model (40). Consensus patterns were derived based upon these metrics, and the scores for the primary pattern (CV1) compared across groups and individuals.

\section{Global Scale Analyses \& Comparison With An External Control Cohort}

Potential effects upon amyloid and FDG PET were additionally evaluated by comparing SUVRs for whole cortex and a temporoparietal ROI (comprising the angular, supramarginal and superior temporal gyri) in the hemisphere of the implant versus the opposite hemisphere and a slightly different set of processing steps described in supplementary materials. This provided a comparator to the standard ROI analyses that had been performed using somewhat different technical approaches and reference regions.

As no control group was recruited in the study, we selected patients from the ADNI database (41). The selection was made from ADNI subjects who had at least two sessions (on average 30 months apart) with T1w MRI, FDG and amyloid PET data, and with an MCl or AD diagnosis at the considered sessions $(42,43)$. The images were processed using the same procedure as the one used for the study subjects but restricted to two time points. A 5:1 patient matching was performed on age, gender and MMSE (caliper =2) using the nearest-neighbor matching method without replacement.

\section{Statistical analysis}

Evolution in regional PET SUVR was tested between M0 and M8 for both the FDG and amyloid tracers using the Wilcoxon signed-rank test. Evolution in neuropsychological scores were compared between M0 and M4; M4 and M8 as well as between M0 and M8, using Wilcoxon signed-rank tests. To correct for multiple testing, we used the Benjamini-Hochberg method. We compared our population to the control ADNI population in terms of demographic characteristics such as age and MMSE and in terms of regional PET SUVR computed at baseline in both the large and small ROIs using Kruskal-Wallis H-test. We also compared the annualized percent change in cognitive and PET SUVR between these two groups. The Benjamini-Hochberg method was also used to correct for multiple testing. All patients with at least one sonication performed were analyzed for efficacy. Safety was described on all included patients. 


\section{Results}

An overview of the trial is shown in Figure 3. Thirteen patients were screened, ten were included and implanted and nine patients completed the trial. The demographics of these patients are shown in Table 1. The patients had mild AD, with a median age of 71 years, 14 years of education and a MMSE of 25 . 
Table 1

Patient Demographics. Notes. Data are given as median [first quartile, third quartile] for continuous variables and as count (percentages) for categorical variables. MMS: Mini mental state, FR: free recall, TR: total recall, Intr: Intrusions, Prax: praxis, FAB: Frontal assessment battery, DelFR: Delayed Free recall, DelTR: Delayed total recall, Cat: categorical, Lit: Litteral, STAl: State-Trait Anxiety Inventory, MADRS: Montgomery-Åsberg Depression Rating Scale, CDR SB: Clinical dementia rating scale sum of boxes, TMT: trail making test

\begin{tabular}{|ll|}
\hline & Patients analysed \\
& $\mathbf{( N = 9 )}$ \\
\hline Gender (Female) & $5(55.6 \%)$ \\
\hline Age (years) & $71.0[69.0,73.0]$ \\
\hline Education (years) & $14.0[12.0,15.0]$ \\
\hline MMS & $25.0[21.0,26.0]$ \\
\hline FR & $4.5[4.0,6.0]$ \\
\hline TR & $11.5[8.75,18.75]$ \\
\hline intr & $18.0[7.0,28.0]$ \\
\hline Rey copy & $29[20.0,32.0]$ \\
\hline Prax & $20.0[17.0,21.0]$ \\
\hline Rey mem & $4[2,6]$ \\
\hline FAB & $14.0[13.0,17.0]$ \\
\hline DelFR & $2.0[0.0,3.0]$ \\
\hline DelTR & $4.0[0.0,11.0]$ \\
\hline Cat Fluency & $17.0[15.0,24.0]$ \\
\hline Lit Fluency & $16.0[13.0,25.0]$ \\
\hline STAl & $49[39,55]$ \\
\hline MADRS & $5.0[5.0,9.0]$ \\
\hline CDR SB & $3.5[2.5,4.0]$ \\
\hline TMT B-A_time & $88.0[61.0,167.5]$ \\
\hline
\end{tabular}


The treated patients received a total of seven sonications each (every two weeks) for a total of 63 sessions to disrupt the BBB using the SonoCloud-1 including nine sessions (1 st sonication in each patient) at $0.9 \mathrm{MPa}$ and 54 sessions at $1.03 \mathrm{MPa}$. The procedure was well-tolerated at both pressure levels used. No patient had redness on the implantation area or pain, no skin infection, no systemic infectious symptom). The time to connection of 4.53 minutes on average (+/- 9.29 minutes), and a median pain evaluation during needle connection of 2 on a scale of 10 were observed in patients.

\section{Safety and Feasibility}

One patient was prematurely withdrawn from the study after no connection was established after two attempts. An MRI revealed a particularly thick scalp in this participant $(8 \mathrm{~mm})$, which led to the connection difficulty and led to a revised non-inclusion criteria to prevent such difficulties in the future.

A total of thirteen AEs (detailed in Table 2) were reported, with all except one event a grade 1. One severe adverse event occurred during the trial. The fourth participant suffered from a delirium for two hours which happened two days after the second BBB opening session. A brain MRI revealed a bleeding on a previously existing microbleed in the left frontal hemisphere, which was $5 \mathrm{~cm}$ from the ultrasound sonication region. An Independent Data Safety Monitoring Board (DSMB) concluded there was an unlikely relation of this $\mathrm{AE}$ to the $\mathrm{BBB}$ opening procedure and the participant agreed to continue sonications on protocol. No subsequent AEs occurred for this participant after this event even after the sonication resumed. 
Table 2

Adverse events description. Treatment-emergent adverse events (CTCAE version number) which occurred during treatment or up to 30 days after the end of therapy. The occurrence of each adverse event is listed as well as the total number of patients affected as some patients might have experienced the same adverse event multiple times over the course of therapy

\begin{tabular}{|llll|}
\hline & $\begin{array}{l}\text { Number of events by } \\
\text { grade }\end{array}$ & $\begin{array}{l}\text { Number (percentage) of } \\
\text { participants }\end{array}$ \\
\hline N=10 patients & Grade 1 & Grade 2 & \\
\hline Delirium with frontal microbleed & & 1 & $1(10 \%)$ \\
\hline Fatigue & 2 & $2(20 \%)$ \\
\hline Back pain & 1 & $1(10 \%)$ \\
\hline Dandruff & 1 & $1(10 \%)$ \\
\hline Prurit & 1 & $1(10 \%)$ \\
\hline Asymptomatic low blood pressure & 1 & $1(10 \%)$ \\
\hline Right hand sensitive deficit & 1 & $1(10 \%)$ \\
\hline $\begin{array}{l}\text { Headaches (intensity range on a visual } \\
\text { scale: 2-4/10) }\end{array}$ & 2 & $2(20 \%)$ \\
\hline Diarrhea & 1 & $1(10 \%)$ \\
\hline Abdominal pain & 2 & $1(20 \%)$ \\
\hline
\end{tabular}

Transdermal needle/implant connection issues were reported for 11 sessions but none of them had any consequences for the patient (no pain or $\mathrm{AE}$ ). In the sessions including an MRI after BBB opening, no immediate AEs were detected radiologically, with no changes in FLAIR or T2* and Diffusion-weighted imaging.

\section{Amyloid PET results}

Each of the nine participants who completed the study had a positive amyloid burden at baseline based upon the visual read of their Florbetapir PET scan as well as measurement of regional SUVRs (Supp Fig. 1). Within the tissue proximal to the SonoCloud-1 implant, 8/9 participants had a positive amyloid burden (AV-45 SUVR referenced to whole cerebellum below 1.1), with patient 10 at the limit of positivity (value=1.11).

Two participants who were amyloid positive in the target region showed evidence of technical artifacts making measurement uncertain. In one participant, the baseline MRI scan had prominent ring artifact characteristic of significant head motion. For these participants, SUVRs in regions unlikely to be affected by sonication exhibited longitudinal change outside of an expected physiologic range. These two subjects were excluded from the results graphs from calculation of amyloid changes in the target region described below. 
SUVRs referenced to a ROI similar to the target ROI but in the opposite hemisphere showed decreases in amyloid in participants with a positive baseline amyloid value and passing quality control. The mean and (S.D.) change from baseline for these seven participants was - $0.73(0.081)$ and $-0.64(0.064)$ for 4- and 8months post baseline, respectively, or $-6.6 \%(7.2 \%)$ and $-5.7 \%(6.2 \%)$ on a percentage basis (Figure 4$)$. Using a similarly sized ROI in the same hemisphere, the mean changes in these participants for 4 and 8 months were $-0.05(0.049)$ and $-0.049(0.044)$ for 4 and 8 months, or $-4.8 \%(4.5 \%)$ and $-4.7 \%(4.1 \%)$. Referenced to white matter, 4 - and 8-months changes were - $0.33(0.041)$ and $-0.015(0.027)$ or $-3.5 \%$ $(4.1 \%)$ and $-1.7 \%(2.8 \%)$; relative to whole cerebellum $-0.249(0.148)$ and $-0.160(0.102)$ or $-13.6 \%(8.6)$ and $-9.2 \%(6.32 \%)$ respectively, and referenced to bilateral parietal cortex $-2.8 \%(2.7 \%)$ and $-1.0 \%(2.2 \%)$. A comparison of left to right large parietal ROI (of which the implant and opposite side ROls were subsets) showed a decrease of $-0.33(0.041)$ and $-0.015(0.027)$ or $-3.5 \%(4.1 \%)$ and $-1.7 \%(2.85)$.

In all cases, a decrease was observed at 4 months that partially but not completely was reduced in magnitude at 8 months (the period during which no sonications were performed). Wilcoxon signed rank test results comparing baseline to 4 months resulted in $p$-values ranging from trend level ( 0.1 , reference adjacent tissue and whole cerebellum) to not significant (other reference regions). For the participant with a sub-threshold implant ROI SUVR (Patient 10), changes ranged from $3 \%$ (referenced to adjacent tissue, white tissue, and bilateral parietal) to $9 \%$ (opposite hemisphere) at 4 months and from $-4 \%$ to $+4 \%$ at 8 months.

\section{FDG PET Results}

The participants exhibited decreases in regional glucose metabolism, referenced to whole cerebellum, in regions consistent with the overall pattern of metabolic decline. A blunting or plateau in decline was observed in the target region referenced to whole cerebellum after four months (Figure 5). When the target region or the broader left supramarginal region was compared to right supramarginal region, a nonsignificant blunting of decline was also observed after four months.

\section{Results of the Global Scale Analyses \& Comparison With An External Control Cohort}

Potential effects of BBB disruption further away from the device, i.e., within the whole cortex and a temporoparietal ROI, were as studied for both amyloid and FDG PET. The amyloid uptake remained on average stable between M0 and M8 (no statistical difference according to the Wilcoxon signed rank test), while the FDG uptake decreased on average between M0 and M8 (significant difference in the left cortex, and in both the left and right temporoparietal ROls according to the Wilcoxon signed rank test after correction for multiple comparisons following the Benjamini-Hochberg procedure), see Supp Table 2.

No statistically significant difference at baseline or in evolution between the subjects that received sonications and the external control group (ADNI) was identified on amyloid, FDG PET nor on cognition (Supp Fig. 2 \& Table 3). 
In the BBB disruption efficacy analysis, post-sonication Gd-DOTA concentration maps were computed from MRI T1 maps, and a sonicated ROI was compared with a non-sonicated control ROI. Detectable ultrasound-mediated BBB disruption was observed for 10/16 (62.5\%) of the sessions with available T1 maps (Table S1). Six of the nine patients (70\%) had detectable BBB disruption for at least one of the two sessions with MR imaging. In the sessions with detected BBB disruption, the average brain Gd-DOTA enhancement volume difference between ROI and control was $0.81 \pm 0.38 \mathrm{~mL}$, and the average difference in Gd-DOTA quantity was $25.7 \pm 8.5 \mu \mathrm{g}$. No significant difference or trend was found when comparing the two sessions with MRI data available (session 1 and session 3 ) for a same patient.

\section{Cognitive change Results}

No statistically significant change was observed on cognitive measures either compared to individual data or to cognitive change evidenced in the matched sample of ADNI participants (Supplementary Fig. 2).

\section{Discussion}

In this study, the feasibility of repeated BBB disruption in early AD patients using an implantable ultrasound device was shown. A total of seven repeated sonication sessions, performed every two weeks, were performed in nine patients. The procedure's tolerance had already been demonstrated in a group of nineteen patients with recurrent glioblastoma that received 65 sonication sessions prior to monthly infusion of carboplatin chemotherapy (median age $=59$ years old) (44). Our study confirms the safety of the device and sonication sessions to disrupt the BBB in a group of older (median age=71 years old) and cognitively impaired individuals. One participant had a delirium that lasted for two hours which occurred two days after a sonication. It was associated with the re-bleeding of a microbleed which was already present prior to study enrolment. This is reminiscent of an amyloid related imaging anomaly of the hemorrhagic type (ARIAh) which is consistent with the trend we observed in amyloid clearance following the seven sonication sessions.

The SonoCloud-1 was implanted at the left parietotemporal junction centered on the left supramarginal gyrus. This tissue was selected because it was accessible (adjacent to skull) for implant placement, is a site of typical AD pathology and decline in glucose metabolism, and is integral to the cognitive decline associated with $A D$. In particular, this region is an associative cerebral region involved in multiple functions including speech, calculation, and gestures, and an improvement in this area could have positive cognitive effects.

Although this was a pilot study, amyloid PET results suggest that SonoCloud- 1 treatment is associated with a trend (not significant) of decreased amyloid burden in tissue proximal to the implant. This decrease was detected using multiple reference regions. These reductions were observed in subjects having a positive baseline amyloid burden in the target region. The lack of decreased signal in the subject with below-threshold amyloid in the target region is consistent with the signal having been associated 
with amyloid rather than a change in local blood flow or technical noise. No effect was observed further away from the region targeted by the implant.

The amyloid reductions observed in our study are consistent with another recent study that used a focused ultrasound system to disrupt the BBB. In that case, the left or right hippocampus was targeted and three sonications were performed every two weeks in six patients (23). A slight reduction was observed between amyloid levels at inclusion versus amyloid levels observed seven days after the third (last) treatment, with a mean reduction of $5 \%$ across patients. No changes were observed in cognitive assessments.

No cognitive effects of sonication were observed, but the study sample size, limited spatial region of sonication, and short follow-up may explain why no variation in cognitive assessments could be demonstrated. Recent large trial data from anti-amyloid immunotherapies indicates that cognitive improvement likely requires more complete removal of amyloid over a longer period of time $(4,45)$. BBB opening using ultrasound coupled with these new antibody treatments could act synergistically and could be explored in further studies. The observation of cognitive improvement over shorter durations in the mouse model (10) may be attributable to the whole hemisphere/brain sonications that were able to be performed, coupled with a more rapid disease or response process.

\section{Limitations}

Limitations of the present work included the limited sample size, study follow-up, and the spatially limited application of sonication. Longer trials may be needed to determine the frequency and duration of sonication required to maintain effect. In addition, other than referencing external studies such as ADNI for comparison of amyloid accumulation rates, there were no controls for the study. As the first study on impaired aged patient, a small sample size was ethically acceptable and given the need for invasive implant placement, it would not have been feasible to have placebo. Despite these limitations, the ability to compare to control regions within-subject provided a basis for preliminary findings regarding effects upon amyloid. The volume of the implant ROI was relatively small and the portion and morphology of gray matter variable, increasing the opportunity for motion induced noise.

\section{Conclusions}

In summary, our results confirm that repeatedly opening the BBB in mild AD patients is both feasible and well tolerated and may be associated with a reduction of amyloid burden. Recent pre-clinical studies also show that ultrasound alone (without microbubbles/BBB disruption) may have positive effects on cognition that we did not evidence in our trial, perhaps due to the sample size (46). These findings as well as the potential for enhanced therapeutic bioavailability in the brain of this approach merit further studies of this new treatment approach for $A D$ and other neurodegenerative diseases.

\section{Abbreviations}


$A D$

Alzheimer's disease

ADNI

Alzheimer's disease neuroimaging initiative

AE

Adverse event

BBB

Blood-brain Barrier

CDR-SB

Clinical dementia rating scale sum of boxes score

CSF

Cerebrospinal fluid

CTCAE

Common terminology criteria for adverse events

FAB

Frontal assessment battery

FCSRT

Free and cued selected reminding test

FDG

Fludeoxyglucose

GBM

Glioblastoma

$\mathrm{Gd}$

Gadolinium

MADRS

Montgomery-Åsberg Depression Rating Scale

$\mathrm{MCl}$

Mild cognitive impairment

MMSE

Mini-mental state examination

$\mathrm{MPa}$

Megapascal

MRI

Magnetic resonance imaging

PET

Positron emission tomography

rGBM

Recurrent glioblastoma

ROI

Region of interest 
SPM

Statistical parametric mapping

STAI

State-trait anxiety inventory

SUV

Standard uptake value

TMT

Trail making test

US

Ultrasound

US-BBBD

Ultrasound-induced blood-brain barrier disruption

\section{Declarations}

\section{Ethics approval and consent to participate}

An Independent Ethics Committee (Comité de Protection des Personnes Ile-de-France VI) approved the protocol on January 10,2017. Informed consent was obtained from all participants and their study partner before inclusion.

\section{Consent for publication}

Not applicable.

\section{Availability of data and materials}

Data is available upon reasonable request to the corresponding author.

\section{Competing interests}

Michael Canney, Carole Desseaux, Guillaume Bouchoux, and Cyril Martin are employees of Carthera and have ownership interest in the company. Alexandre Carpentier is a paid consultant to Carthera and has ownership interest in Carthera.

\section{Funding}

This project was realized with the support of BPI France. SonoCloud-1 devices and technical support was supplied by Carthera (Paris, France).

\section{Authors' contributions}

Stéphane Epelbaum (SE) and Alexandre Carpentier (AC) contributed to the study concept, design, and writing of the manuscript. Ninon Burgos, Michael Canney, Dawn Matthews, Guillaume Bouchoux, Lisa 
Belin, SE, and AC contributed to the writing of the manuscript, acquisition of the data, and analysis. AC performed the implantation of the SonoCloud. Cyril Martin assisted with sonications. All authors read and approved the final manuscript.

\section{Acknowledgements}

The sponsor of the study was Assistance Publique - Hôpitaux de Paris (Délégation à la Recherche Clinique et à l'Innovation). The authors acknowledge the help of the independent monitoring committee, Dr Stéphane Haïk, Julien Savatovsky and Nadine Petipain, of the Délégation à la Recherche Clinique et à I'Innovation, notably Moufida Dabbech and Hakim Souag, the Vigilance unit and clinical research unit of the Pitié Salpêtrière hospital as well as the patients and their family for their support and participation during this trial.

Data collection and sharing for this project was funded by the Alzheimer's Disease Neuroimaging Initiative (ADNI) (National Institutes of Health Grant U01 AG024904) and DOD ADNI (Department of Defense award number W81XWH-12-2-0012). ADNI is funded by the National Institute on Aging, the National Institute of Biomedical Imaging and Bioengineering, and through generous contributions from the following: AbbVie, Alzheimer's Association; Alzheimer's Drug Discovery Foundation; Araclon Biotech; BioClinica, Inc.; Biogen; Bristol-Myers Squibb Company; CereSpir, Inc.; Cogstate; Eisai Inc.; Elan Pharmaceuticals, Inc.; Eli Lilly and Company; Eurolmmun; F. Hoffmann-La Roche Ltd and its affiliated company Genentech, Inc.; Fujirebio; GE Healthcare; IXICO Ltd.; Janssen Alzheimer Immunotherapy Research \& Development, LLC.; Johnson \& Johnson Pharmaceutical Research \& Development LLC.; Lumosity; Lundbeck; Merck \& Co., Inc.; Meso Scale Diagnostics, LLC.; NeuroRx Research; Neurotrack Technologies; Novartis Pharmaceuticals Corporation; Pfizer Inc.; Piramal Imaging; Servier; Takeda Pharmaceutical Company; and Transition Therapeutics. The Canadian Institutes of Health Research is providing funds to support ADNI clinical sites in Canada. Private sector contributions are facilitated by the Foundation for the National Institutes of Health (www.fnih.org). The grantee organization is the Northern California Institute for Research and Education, and the study is coordinated by the Alzheimer's Therapeutic Research Institute at the University of Southern California. ADNI data are disseminated by the Laboratory for Neuro Imaging at the University of Southern California.

\section{References}

1. Cummings J, Lee G, Ritter A, Sabbagh M, Zhong K. Alzheimer's disease drug development pipeline: 2020. Alzheimer's Dement Transl Res Clin Interv. 2020;6(1):e12050.

2. Howard R, Liu KY. Questions EMERGE as Biogen claims aducanumab turnaround. Nat Rev Neurol. 2020;16(2):63-4.

3. Schneider L. A resurrection of aducanumab for Alzheimer's disease. Lancet Neurol. 2020;19(2):1112.

4. Sevigny J, Chiao P, Bussière T, Weinreb $P H$, Williams L, Maier $M$, et al. The antibody aducanumab reduces A\$ $\beta \$$ plaques in Alzheimer's disease. Nature. 2016;537(7618):50-6. 
5. Banks WA, Terrell B, Farr SA, Robinson SM, Nonaka N, Morley JE. Passage of amyloid $\beta$ protein antibody across the blood-brain barrier in a mouse model of Alzheimer's disease. Peptides. 2002;23(12):2223-6.

6. Pardridge WM. Targeted delivery of protein and gene medicines through the blood-brain barrier. Clin Pharmacol Ther. 2015 Apr;97(4):347-61.

7. Hynynen K, McDannold N, Vykhodtseva N, Jolesz FA. Noninvasive MR Imaging-guided Focal Opening of the Blood-Brain Barrier in Rabbits. Radiology. 2001;220(3):640-6.

8. Dréan A, Lemaire N, Bouchoux G, Goldwirt L, Canney M, Goli L, et al. Temporary blood-brain barrier disruption by low intensity pulsed ultrasound increases carboplatin delivery and efficacy in preclinical models of glioblastoma. J Neurooncol. 2019;144(1).

9. Zhang DY, Dmello C, Chen L, Arrieta VA, Gonzalez-Buendia E, Robert Kane J, et al. Ultrasoundmediated delivery of paclitaxel for glioma: A comparative study of distribution, toxicity, and efficacy of albumin-bound versus cremophor formulations. Clin Cancer Res [Internet]. 2020 Dec 12;26(2):477-86. Available from:

http://clincancerres.aacrjournals.org/content/early/2019/12/11/1078-0432.CCR-19-2182.abstract

10. Leinenga G, Koh WK, Götz J. A comparative study of the effects of Aducanumab and scanning ultrasound on amyloid plaques and behavior in the APP23 mouse model of Alzheimer disease. bioRxiv. 2021;2001-21.

11. Jordão JF, Thévenot E, Markham-Coultes K, Scarcelli T, Weng YQ, Xhima K, et al. Amyloid- $\beta$ plaque reduction, endogenous antibody delivery and glial activation by brain-targeted, transcranial focused ultrasound. Exp Neurol. 2013;

12. Pandit R, Leinenga G, Götz J. Repeated ultrasound treatment of tau transgenic mice clears neuronal tau by autophagy and improves behavioral functions. Theranostics. 2019;

13. Poon C, Pellow C, Hynynen K. Neutrophil recruitment and leukocyte response following focused ultrasound and microbubble mediated blood-brain barrier treatments. Theranostics. 2021;11(4):1655.

14. Kinoshita M, McDannold N, Jolesz FA, Hynynen K. Targeted delivery of antibodies through the blood-brain barrier by MRI-guided focused ultrasound. Biochem Biophys Res Commun. 2006;340(4):108590.

15. Alkins R, Burgess A, Ganguly M, Francia G, Kerbel R, Wels WS, et al. Focused ultrasound delivers targeted immune cells to metastatic brain tumors. Cancer Res. 2013;73(6):1892-9.

16. Mainprize T, Lipsman N, Huang $Y$, Meng $Y$, Bethune A, Ironside $S$, et al. Blood-Brain Barrier Opening in Primary Brain Tumors with Non-invasive MR-Guided Focused Ultrasound: A Clinical Safety and Feasibility Study. Sci Rep. 2019;9(1):321.

17. Carpentier A, Canney M, Vignot A, Reina V, Beccaria K, Horodyckid C, et al. Clinical trial of blood-brain barrier disruption by pulsed ultrasound. Sci Transl Med. 2016;8(343):343re2.

18. Idbaih A, Canney M, Belin L, Desseaux C, Vignot A, Bouchoux G, et al. Safety and feasibility of repeated and transient blood-brain barrier disruption by pulsed ultrasound in patients with recurrent 
glioblastoma. Clin Cancer Res. 2019;25(13).

19. Park SH, Kim MJ, Jung HH, Chang WS, Choi HS, Rachmilevitch I, et al. One-Year Outcome of Multiple Blood-Brain Barrier Disruptions With Temozolomide for the Treatment of Glioblastoma. Front Oncol. 2020;10:1663.

20. Rezai AR, Ranjan M, D'Haese PF, Haut MW, Carpenter J, Najib U, et al. Noninvasive hippocampal blood-brain barrier opening in Alzheimer's disease with focused ultrasound. Proc Natl Acad Sci U S A [Internet]. 2020 Apr 28 [cited 2020 Oct 26];117(17):9180-2. Available from: https://github.com/pd0033/PNAS-FUS.git

21. Abrahao A, Meng Y, Llinas M, Huang Y, Hamani C, Mainprize T, et al. First-in-human trial of bloodbrain barrier opening in amyotrophic lateral sclerosis using MR-guided focused ultrasound. Nat Commun. 2019;10(1):1-9.

22. Rezai AR, Ranjan M, D'Haese P-F, Haut MW, Carpenter J, Najib U, et al. Noninvasive hippocampal blood- brain barrier opening in Alzheimer's disease with focused ultrasound. Proc Natl Acad Sci. 2020;117(17):9180-2.

23. D'Haese P-F, Ranjan M, Song A, Haut MW, Carpenter J, Dieb G, et al. $\beta$-Amyloid Plaque Reduction in the Hippocampus After Focused Ultrasound-Induced Blood-Brain Barrier Opening in Alzheimer's Disease. Front Hum Neurosci. 2020;14:422.

24. Dubois B, Feldman HH, Jacova C, Hampel H, Molinuevo JL, Blennow K, et al. Advancing research diagnostic criteria for Alzheimer's disease: the IWG-2 criteria. Lancet Neurol. 2014;13(6):614-29.

25. Teichmann M, Epelbaum S, Samri D, Nogueira ML, Michon A, Hampel H, et al. Free and Cued Selective Reminding Test-accuracy for the differential diagnosis of Alzheimer's and neurodegenerative diseases: a large-scale biomarker-characterized monocenter cohort study (ClinAD). Alzheimer's Dement. 2017;13(8):913-23.

26. Folstein MF, Folstein SE, McHugh PR. "Mini-mental state": a practical method for grading the cognitive state of patients for the clinician. J Psychiatr Res. 1975;12(3):189-98.

27. Berg L, Miller JP, Baty J, Rubin EH, Morris JC, Figiel G. Mild senile dementia of the Alzheimer type. 4. Evaluation of intervention. Ann Neurol Off J Am Neurol Assoc Child Neurol Soc. 1992;31(3):242-9.

28. O'Bryant SE, Waring SC, Cullum CM, Hall J, Lacritz L, Massman PJ, et al. Staging dementia using Clinical Dementia Rating Scale Sum of Boxes scores: a Texas Alzheimer's research consortium study. Arch Neurol. 2008;65(8):1091-5.

29. Dubois B, Slachevsky A, Litvan I, Pillon B. The FAB: a frontal assessment battery at bedside. Neurology. 2000;55(11):1621-6.

30. Grober E, Buschke H, Crystal H, Bang S, Dresner R. Screening for dementia by memory testing. Neurology. 1988;38(6):900.

31. Reitan RM. Validity of the Trail Making Test as an indicator of organic brain damage. Percept Mot Skills. 1958;8(3):271-6.

32. Cardebat D, Doyon B, Puel M, Goulet P, Joanette Y. Formal and semantic lexical evocation in normal subjects. Performance and dynamics of production as a function of sex, age and educational level. 
Acta Neurol Belg. 1990;90(4):207-17.

33. Mahieux-Laurent F, Fabre C, Galbrun E, Dubrulle A, Moroni C. Validation of a brief screening scale evaluating praxic abilities for use in memory clinics. Evaluation in 419 controls, 127 mild cognitive impairment and 320 demented patients. Rev Neurol (Paris). 2009;165(6-7):560-7.

34. Rey A. L'examen psychologique dans les cas d'encéphalopathie traumatique.(Les problems.). Arch Psychol (Geneve). 1941;

35. Spielberger CD. Manual for the State-Trait Anxiety Inven- tory: STAI (Form Y). Palo Alto, CA: Consulting Psychologists Press; 1983.

36. Montgomery S, Asberg M. A New Depression Scale Designed to be Sensitive to Change. $\mathrm{Br} \mathrm{J}$ Psychiatry. 1979;134(4):382-9.

37. Wiesinger F, Sacolick LI, Menini A, Kaushik SS, Ahn S, Veit-Haibach P, et al. Zero TE MR bone imaging in the head. Magn Reson Med. 2016;75(1):107-14.

38. Shen Y, Goerner FL, Snyder C, Morelli JN, Hao D, Hu D, et al. T1 relaxivities of gadolinium-based magnetic resonance contrast agents in human whole blood at 1.5, 3, and $7 \mathrm{~T}$. Invest Radiol. 2015;50(5):330-8.

39. Chen K, Roontiva A, Thiyyagura P, Lee W, Liu X, Ayutyanont N, et al. Improved power for characterizing longitudinal amyloid- $\beta$ PET changes and evaluating amyloid-modifying treatments with a cerebral white matter reference region. J Nucl Med. 2015;56(4):560-6.

40. Strother S, Oder A, Spring R, Grady C. The NPAIRS computational statistics framework for data analysis in neuroimaging. In: Proceedings of COMPSTAT'2010. Springer; 2010. p. 111-20.

41. Spiegel R, Berres M, Miserez AR, Monsch AU. For debate: substituting placebo controls in long-term Alzheimer's prevention trials. Alzheimers Res Ther. 2011;3(2):1-11.

42. Jack Jr CR, Barnes J, Bernstein MA, Borowski BJ, Brewer J, Clegg S, et al. Magnetic resonance imaging in Alzheimer's disease neuroimaging initiative 2. Alzheimer's Dement. 2015;11(7):740-56.

43. Jagust WJ, Landau SM, Koeppe RA, Reiman EM, Chen K, Mathis CA, et al. The Alzheimer's disease neuroimaging initiative 2 PET core: 2015. Alzheimer's Dement. 2015;11(7):757-71.

44. Dréan A, Lemaire N, Bouchoux G, Goldwirt L, Canney M, Goli L, et al. Temporary blood-brain barrier disruption by low intensity pulsed ultrasound increases carboplatin delivery and efficacy in preclinical models of glioblastoma. J Neurooncol. 2019;

45. Mintun MA, Lo AC, Duggan Evans C, Wessels AM, Ardayfio PA, Andersen SW, et al. Donanemab in early Alzheimer's disease. N Engl J Med. 2021;384(18):1691-704.

46. Blackmore DG, Turpin F, Palliyaguru T, Evans HT, Chicoteau A, Lee W, et al. Low-intensity ultrasound restores long-term potentiation and memory in senescent mice through pleiotropic mechanisms including NMDAR signaling. Mol Psychiatry. 2021;1-17.

47. Tustison NJ, Avants BB, Cook PA, Zheng Y, Egan A, Yushkevich PA, et al. N4ITK: improved N3 bias correction. IEEE Trans Med Imaging. 2010;29(6):1310-20. 
48. Modat M, Cash DM, Daga P, Winston GP, Duncan JS, Ourselin S. Global image registration using a symmetric block-matching approach. J Med Imaging. 2014;1(2):24003.

49. Cardoso MJ, Modat M, Wolz R, Melbourne A, Cash D, Rueckert D, et al. Geodesic information flows: spatially-variant graphs and their application to segmentation and fusion. IEEE Trans Med Imaging. 2015;34(9):1976-88.

50. Rasmussen JM, Lakatos A, van Erp TGM, Kruggel F, Keator DB, Fallon JT, et al. Empirical derivation of the reference region for computing diagnostic sensitive 18fluorodeoxyglucose ratios in Alzheimer's disease based on the ADNI sample. Biochim Biophys Acta (BBA)-Molecular Basis Dis. 2012;1822(3):457-66.

51. Landau SM, Fero A, Baker SL, Koeppe R, Mintun M, Chen K, et al. Measurement of longitudinal $\beta$ amyloid change with 18F-florbetapir PET and standardized uptake value ratios. J Nucl Med. 2015;56(4):567-74.

\section{Figures}




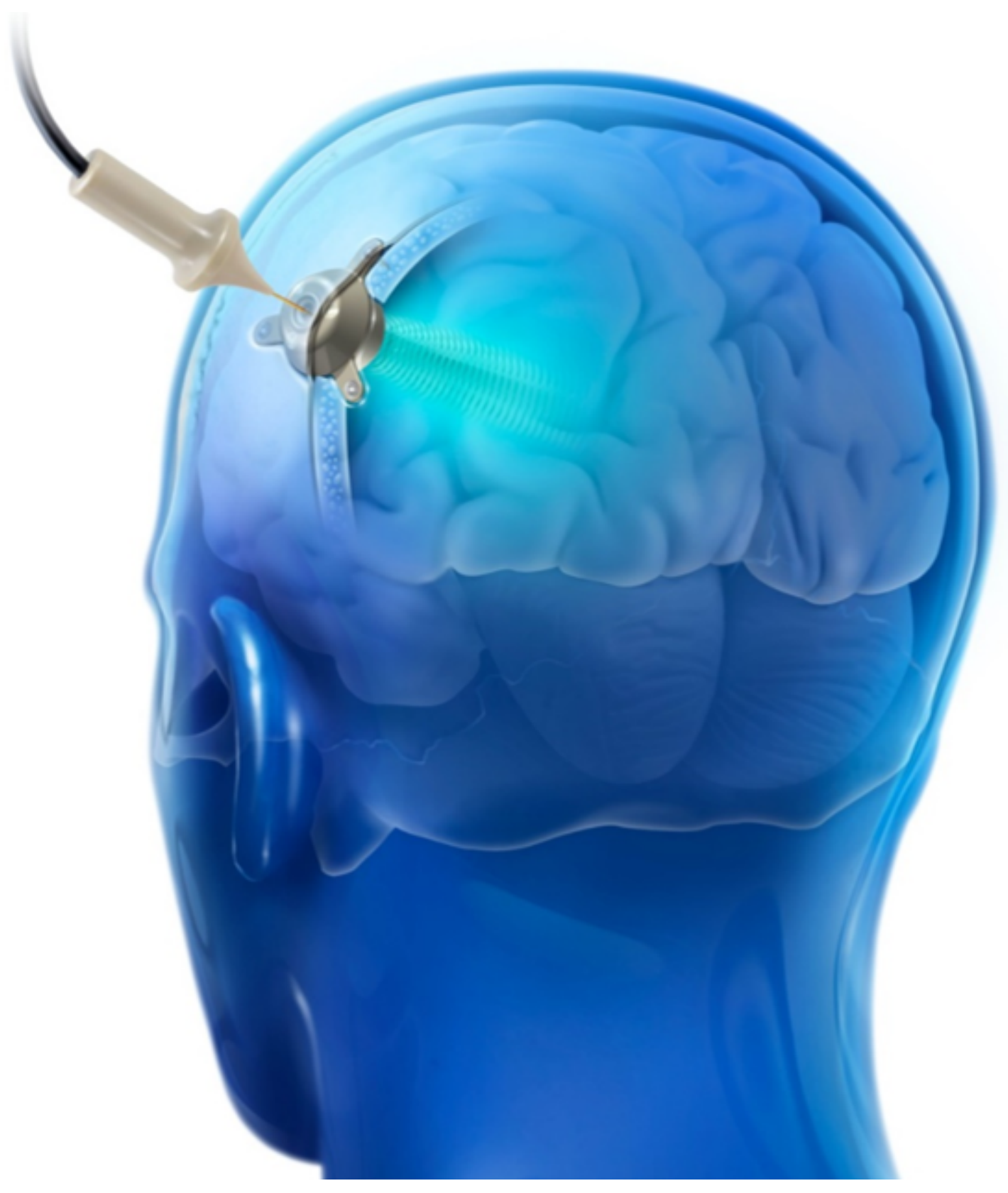

\section{Figure 1}

The SonoCloud-1 is a $1 \mathrm{MHz}$ implantable ultrasound device that is implanted extradurally within the skull thickness and activated at each treatment cycle by connecting it using a transdermal needle to an external radiofrequency generator. In this study, the SonoCloud-1 was implanted to target and temporarily disrupt the BBB above the left supramarginal gyrus. 


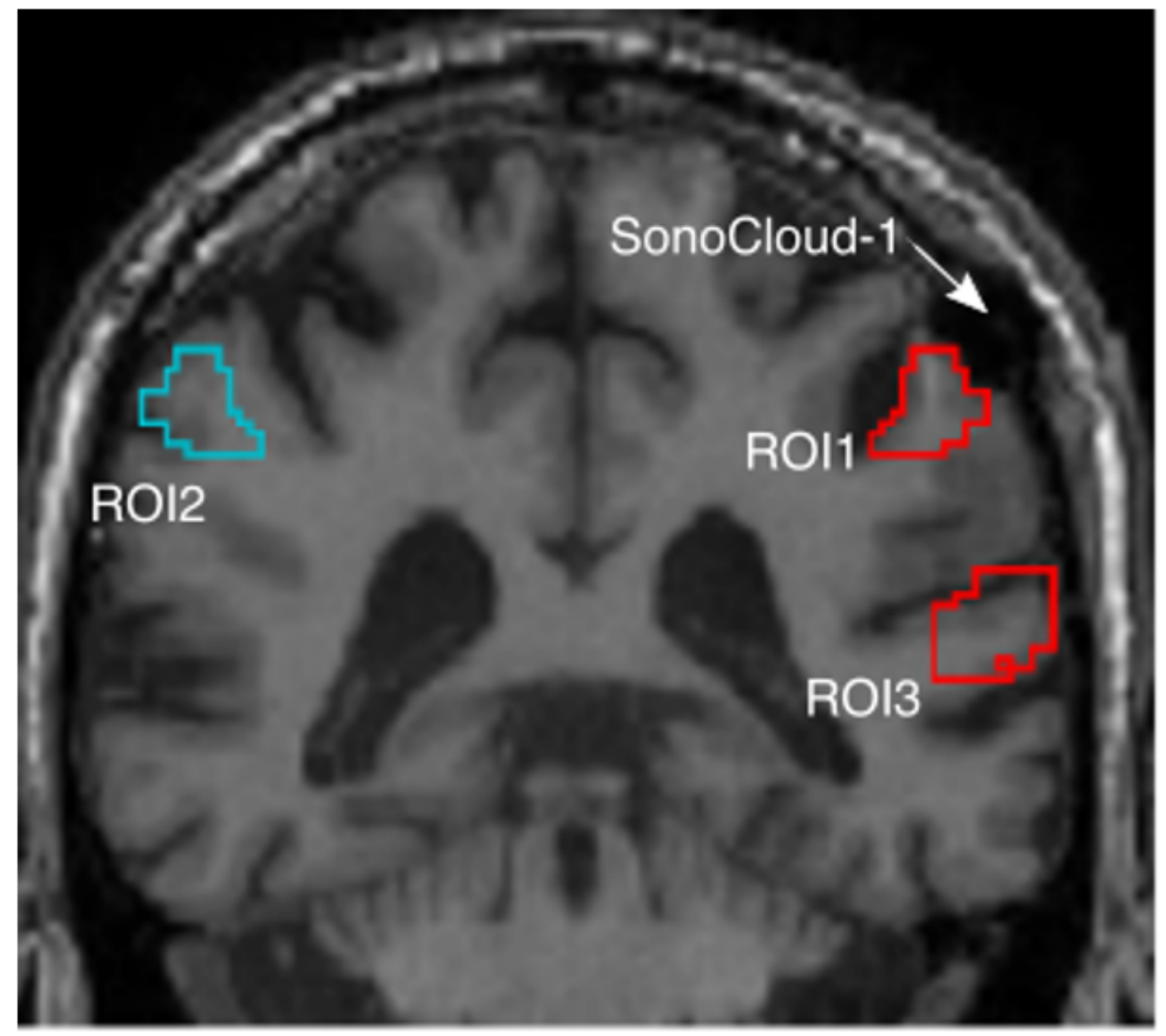

\section{Figure 2}

Region of interest (ROI) definition to measure changes in PET amyloid levels in gray matter targeted by the implant (ROI1) as compared to similar tissue in the opposite (ROI2) and same hemispheres (ROI3). 

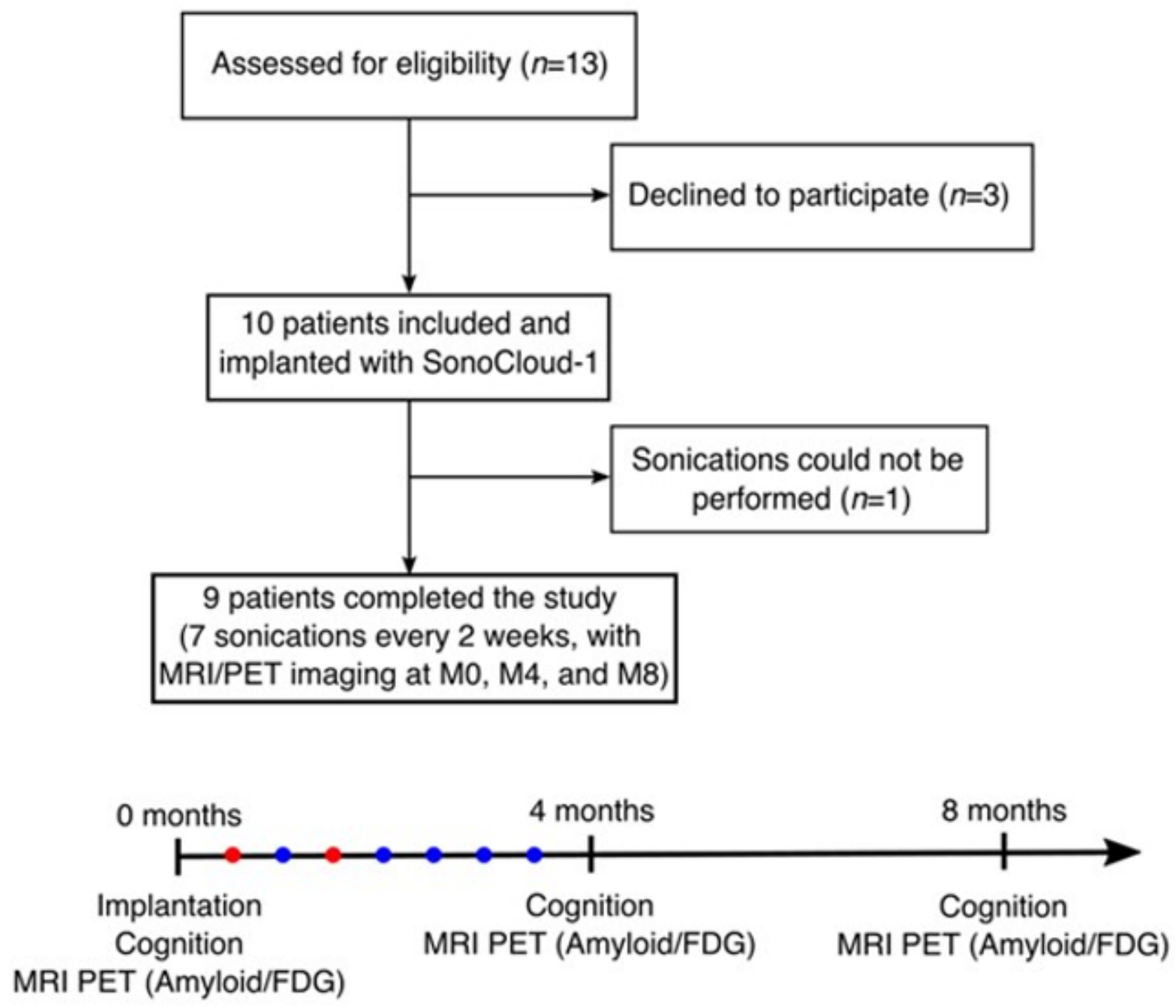

Figure 3

Trial Overview 


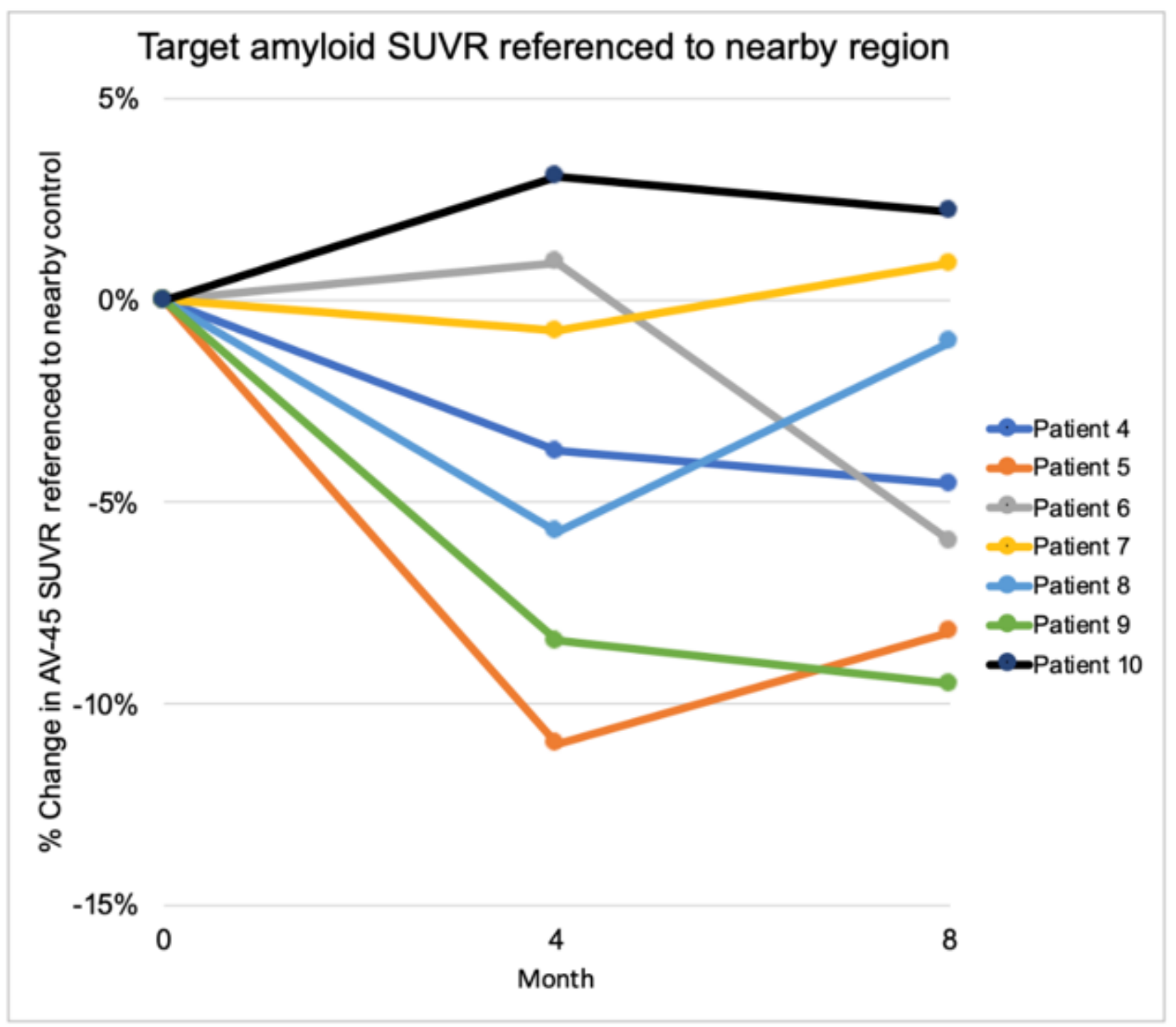

Figure 4

PET Amyloid (florbetapir) change observed in region targeted by SonoCloud-1 device (ROI1). SUVRs referenced to a ROI similar to the target ROI but in the opposite hemisphere (ROI2) showed decreases from baseline of $-6.6 \%(S D=7.2 \%)$ at four months and $-5.7 \%(S D=6.2 \%)$ at eight months. 


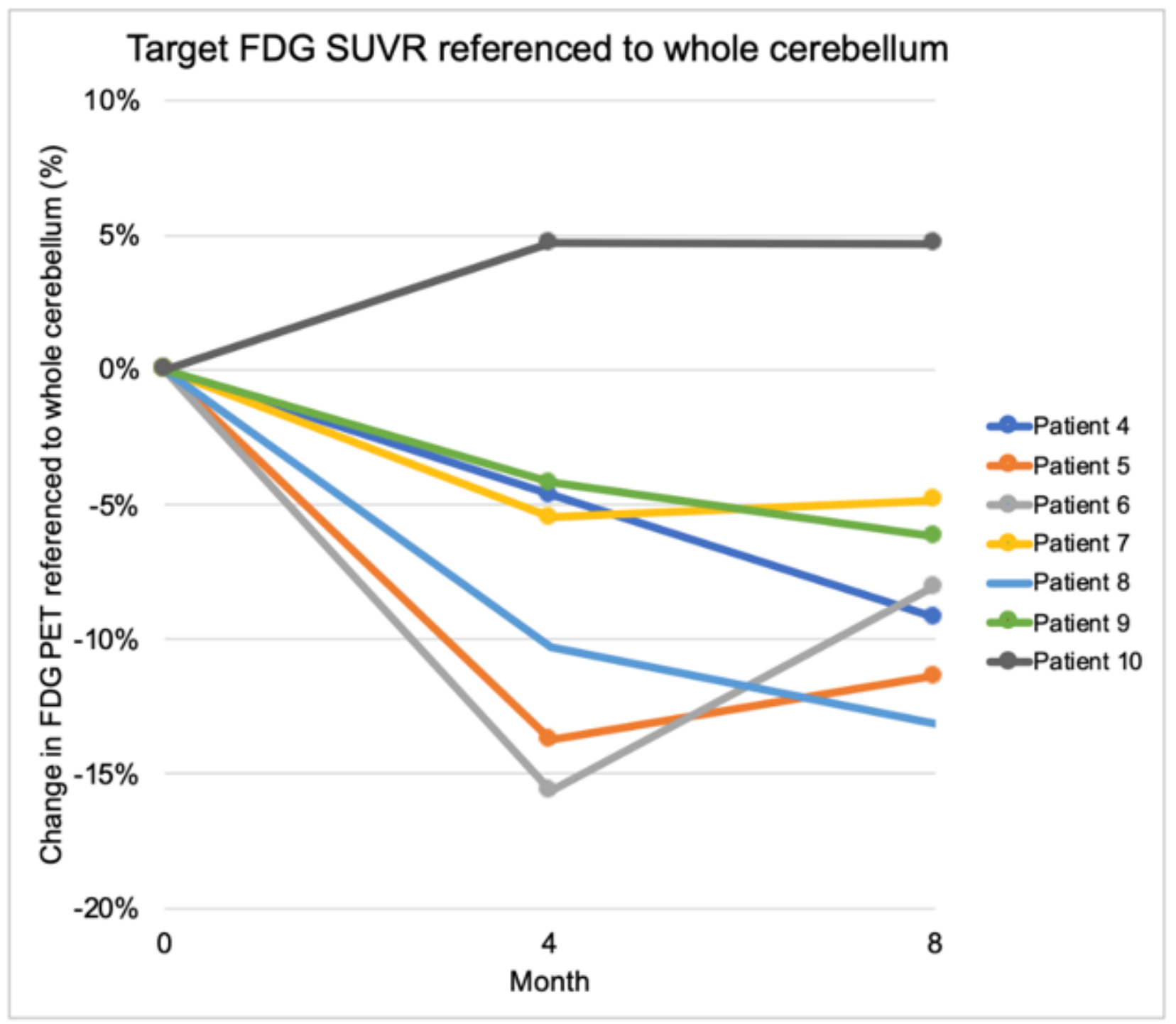

Figure 5

FDG PET changes in target ROI (ROI1) referenced to whole cerebellum. Overall, a blunting or plateau of decline was observed in the target region referenced to whole cerebellum after four months.

\section{Supplementary Files}

This is a list of supplementary files associated with this preprint. Click to download.

- SUPPLEMENTARYMATERIALS.docx 